\title{
NEIL3 contributes toward the carcinogenesis of liver cancer and regulates PI3K/Akt/mTOR signaling
}

\author{
WEICHEN WANG ${ }^{1}$, QING YIN ${ }^{2}$, SHANSHAN GUO $^{3}$ and JUN WANG ${ }^{1}$ \\ ${ }^{1}$ Medical Research and Laboratory Diagnostic Center and ${ }^{2}$ Department of Medical Education, \\ Jinan Central Hospital Affiliated to Shandong First Medical University, Jinan, Shandong 250013; \\ ${ }^{3}$ Department of Food Science and Nutrition, University of Jinan, Jinan, Shandong 250022, P.R. China
}

Received May 12, 2020; Accepted February 12, 2021

DOI: $10.3892 / \mathrm{etm} .2021 .10487$

\begin{abstract}
Liver cancer is one of the top three fatal types of cancer and it causes several thousands of mortalities each year. The main treatment is surgical resection which shows little benefit for patients with recurrence or metastasis. NEIL3 promotes progression and predicts survival in cancer. However, its role in liver cancer remains unclear. Based on data in the TCGA database, NEIL3 exhibited much higher expression in liver cancer tissues and was clinically correlated with tumor grade in patients with liver cancer. Furthermore, high NEIL3 expression caused shorter survival times. In liver cancer cell lines, NEIL3 showed abundant expression. When NEIL3 was knocked down in HepG2 and Huh-7 cells, cell abilities including proliferation, growth, migration and invasion, exhibited deficiency to different extents. Cell cycle transition was blocked at the G2 phase and the cell apoptotic rate increased notably. In addition, the phosphorylation levels of Akt, PI3K and mTOR were increased following NEIL3-overexpression but decreased following NEIL3-knockdown. In conclusion, NEIL3 contributes toward development and/or progression in liver cancer and regulates $\mathrm{PI} 3 \mathrm{~K} / \mathrm{Akt} / \mathrm{mTOR}$ signaling.
\end{abstract}

\section{Introduction}

Liver cancer is a great threat to people's lives. Over 30,000 people die of liver and intrahepatic bile duct cancer each year, with more than 40,000 new patients annually (1). As a great killer, the major therapies, including surgical resection, chemotherapy and radiotherapy are not effective for all patients, particularly those with recurrence or distant metastasis (2). Approximately $18 \%$ of patients are diagnosed with distant

Correspondence to: Dr Jun Wang, Medical Research and Laboratory Diagnostic Center, Jinan Central Hospital Affiliated to Shandong First Medical University, 105 Jiefang Road, Jinan, Shandong 250013, P.R. China

E-mail: 18764116600@163.com

Key words: NEIL3, PI3K, Akt, mTOR, liver cancer metastasis and $27 \%$ with regional metastasis (1). Only $44 \%$ of patients are diagnosed at the localized stage. As a result, the total five-year survival rate for patients with liver cancer is just $18 \%$ (1). In fact, the prognosis for patients with liver cancer is disappointing and requires further investigation.

Lack of knowledge regarding the mechanism underlying liver cancer is one of the major reasons causing its poor clinical outcome. In addition, heterogeneity further increases the complexity and difficulty involved in curing patients with liver cancer (3). By analyzing the reports from the PubMed database, a list of genes contributing toward the development and progression of liver cancer may be constructed. Among them, some are dominant genes, while others serve auxiliary function. Due to heterogeneity, the dominant gene is different in a particular cohort of patients, leading toward different responses to specialized drugs $(3,4)$. For example, sorafenib is a small molecular inhibitor targeting Raf-1 and has been applied in the clinic for the treatment of recurrent or metastatic liver cancer (5). However, resistance to sorafenib has been reported extensively and poses a novel challenge to patients with liver cancer (6). In fact, application of sorafenib in clinics is limited greatly in certain patients. Therefore, it is very important to investigate the molecular mechanism underlying liver cancer.

NEIL3, also called nei-like DNA glycosylase 3, is mapped at chromosome $4 \mathrm{q} 34.3$ and encodes a protein belonging to the DNA glycosylase family (7). NEIL3 was reported to initiate the DNA base excision repair process and cleave the bases damaged by radiation or oxidative response (7-9). NEIL3 has served important roles in brain development and neurogenesis or protection (10-12). Additionally, Skarpengland et al (13) reported that NEIL3 regulated lipid metabolism and prevented atherosclerosis in mice. NEIL3 is also involved in the progression of cerebral ischemia, autoimmunity, Huntington's disease and HIV replication (14-17). NEIL3 also serves important roles in cancer (18). For example, Kim et al (19) reported that polymorphisms in NEIL3 increased the risk of prostate cancer. In glioblastoma, loss of NEIL3 increased replication-associated double strand breaks in DNA strands (20). Aberration in NEIL3 was associated with short survival times in patients with colorectal cancer and astrocytoma $(21,22)$. In breast, ovarian and prostate cancer, NEIL3 affected cancer progression (23-25). NEIL3 may repair telomere damage during 
the mitosis process (26). Telomeres are critical components in the maintenance of cell life and maybe candidate targets in cancer therapy $(27,28)$. Using bioinformatics analysis, Zhang et al (29) identified SNPs in NEIL3 in liver cancer. However, no additional data regarding the role of NEIL3 in liver cancer was found.

The present study aimed to investigate the role of NEIL3 in liver cancer through decreasing the expression of NEIL3 in HepG 2 cells. Next, cell growth, proliferation, migration, invasion, cycle transition and apoptosis were analyzed. In addition, the clinical value of NEIL3 in liver cancer and the preliminary molecular mechanism was investigated.

\section{Materials and methods}

Cell lines and cell culture. Human liver cancer HepG2 and Huh-7cells were purchased from American Type Cell Culture collection and cultured in Dulbecco's modified Eagle's medium (DMEM; Gibco; Thermo Fisher Scientific, Inc.) containing 10\% FBS (Gibco; Thermo Fisher Scientific, Inc.) and 1\% antibiotics (penicillin and streptomycin) in an atmosphere with $5 \% \mathrm{CO}_{2}$ at $37^{\circ} \mathrm{C}$. All cells had been authenticated using the STR method (30).

Reverse transcription-quantitative polymerase chain reaction $(R T-q P C R)$. Total RNA was extracted from cancer cell lines using RNAeasy ${ }^{\mathrm{TM}}$ kit (Beyotime Institute of Biotechnology), according to the manufacturer's protocols, and the concentration of RNA was determined on an ultraviolet spectrophotometer (NanoDrop 2000; Thermo Fisher Scientific, Inc.). Next, $1 \mu \mathrm{g}$ RNA was reverse transcribed $\left(42^{\circ} \mathrm{C}\right.$ for $\left.50 \mathrm{~min}\right)$ into first-strand cDNA using the BeyoRT ${ }^{\mathrm{TM}}$ III cDNA kit (Beyotime Institute of Biotechnology). Next, qPCR was performed on an ABI7000 (Applied Biosystems; Thermo Fisher Scientific, Inc.) using SYBR-Green qPCR mix (Shanghai Yeasen Biotechnology Co., Ltd.). $\beta$-actin was the internal control. The primers for qPCR are listed in Table I. The thermocycling conditions were as follows: $95^{\circ} \mathrm{C}$ for $2 \mathrm{~min}$; $\left(95^{\circ} \mathrm{C}\right.$ for $10 \mathrm{sec} ; 60^{\circ} \mathrm{C}$ for $\left.15 \mathrm{sec}\right)$ for 40 cycles. The relative mRNA levels of target genes were calculated using the $2^{-\Delta \Delta \mathrm{Cq}}$ method (31).

Western blotting. Total proteins were extracted from cancer cells using a protein extraction kit (Beyotime Institute of Biotechnology), according to the manufacturer's protocols. Next, equal amounts of $10 \mu \mathrm{g}$ of protein were analyzed by $12 \%$ SDS-PAGE and transferred onto PVDF membranes (Beyotime Institute of Biotechnology). Following blocking with $5 \%$ skimmed milk for $1 \mathrm{~h}$ at room temperature, primary antibodies against phosphorylated PI3K (cat. no. ab138364; dilution, 1:1,000; Abcam), phosphorylated mTOR (cat. no. ab109268; dilution, 1:2,500; Abcam), phosphorylated Akt (cat. no. ab38449; dilution, 1:500; Abcam) and GAPDH(cat. no. ab181602; dilution, 1:10,000; Abcam) were added to the vessel and co-incubated overnight at $4^{\circ} \mathrm{C}$. Subsequently, the PVDF membranes were washed with PBS (Beyotime Institute of Biotechnology) and incubated with HRP-conjugated goat anti-rabbit IgG antibody (cat. no. ab7090; dilution, 1:5,000; Abcam) for $2 \mathrm{~h}$ at room temperature. Finally, target proteins were detected using chemiluminescence assay kits (Beyotime Institute of Biotechnology) and images were captured.

Construction of the expression plasmid, pcDNA3.1-NEIL3, siRNA synthesis and transfection. The human NEIL3 gene was searched in the NCBI-gene database (https://www.ncbi. nlm.nih.gov/nuccore/NM_018248.3), synthesized and cloned into the expression plasmid, pcDNA3.1. Next, the recombinant expression plasmid, pcDNA3.1-oeNEIL3 (oeNEIL3), was confirmed by DNA sequencing. siRNA oligonucleotide targeting the human NEIL3 gene (siNEIL3) was designed and synthesized. A random sequence was used as negative control. Next, Lipofectamine 3000 reagent (Invitrogen; Thermo fisher Scientific, Inc.) was used to transfectsi NEIL3 (\#1forward, GAGCAGAAAGUGAAGUUAATT and reverse, UUAACUUCACUUUCUGCUCTT; \#2 forward, GCUCAA GAGUGAAGAAAAUTT and reverse, AUUUUCUUCACU CUUGAGCTT) or oeNEIL3 (sequence listed on https://www. ncbi.nlm.nih.gov/nuccore/NM_018248.3) into HepG2 cancer cells. In brief, 50 pmol siNEIL 3 or $0.2 \mu \mathrm{g}$ oeNEIL 3 with $1 \mu \mathrm{l}$ Lipofectamine 3000 was mixed for $20 \mathrm{~min}$ and co-cultured with HepG2 cancer cells for $6 \mathrm{~h}$ at $37^{\circ} \mathrm{C}$. Next, the supernatant was replaced with fresh DMEM and cultured at $37^{\circ} \mathrm{C}$ with $5 \% \mathrm{CO}_{2}$. The time interval between transfection and subsequent experiment was $48 \mathrm{~h}$.

Cell Counting kit-8 (CCK-8). HepG2 cells treated with siNEIL3 or scramble were seeded into a 96 -well plate at $3 \times 10^{3}$ cells/well and cultured for 4 days. At designated time points of 24, 48, 72 and 96 h, $10 \mu \mathrm{l} \mathrm{CCK-8} \mathrm{(Beyotime} \mathrm{Institute} \mathrm{of} \mathrm{Biotechnology)}$ was added, and cells were cultured for another $2 \mathrm{~h}$. The absorbance value was determined at $450 \mathrm{~nm}$ wavelength using a microplate reader (Bio-Rad Laboratories, Inc.).

Plate-colony formation assay. HepG2 cells treated with siNEIL3 or scramble were seeded onto 24 -well plates at $1 \times 10^{3}$ cells/well and cultured for ten days at $37^{\circ} \mathrm{C}$ in a humid environment with $5 \% \mathrm{CO}_{2}$. Next, cell colonies were fixed in $4 \%$ paraformaldehyde (Beyotime Institute of Biotechnology) at room temperature for $30 \mathrm{~min}$, washed with cold PBS, and stained with $0.5 \%$ crystal violet (Beyotime Institute of Biotechnology) at room temperature for $15 \mathrm{~min}$. Next, positively-stained cancer cells were counted under a light inverted microscope (NikonTS100; Nikon Corporation) at x100 magnification. The relative colony formation ability was evaluated by counting the positively-stained clones in each well and the number of clones was presented as a bar graph.

Wound-healing assay. A total of $2 \times 10^{5}$ siNEIL3- or scramble-treated cells per well were seeded onto 24-well plates and cultured for $24 \mathrm{~h}$. When cell confluence arrived at $90 \%$, a scratch was produced by a $10 \mu 1$ sterile tip. The debris was washed gently, and the width of each scratch was recorded and set as the $0 \mathrm{~h}$ time point $\left(\mathrm{W}_{0 \mathrm{~h}}\right)$. Next, fresh DMEM with no serum was added and cells were cultured for an additional $24 \mathrm{~h}$. Subsequently, the width of each scratch was recorded and set as the $24 \mathrm{~h}$ time point $\left(\mathrm{W}_{24 \mathrm{~h}}\right)$. The relative migration rate was detected under a light inverted microscope (NikonTS100; Nikon Corporation) at x100 magnification and calculated as follows: $\mathrm{R}=\left(\mathrm{W}_{24 \mathrm{~h}}-\mathrm{W}_{0 \mathrm{~h}}\right) / \mathrm{W}_{0 \mathrm{~h}}$. 
Table I. Primers for quantitative polymerase chain reaction amplification of NEIL3 and $\beta$-actin.

\begin{tabular}{lll}
\hline Gene & & Primer sequence (5'-3') \\
\hline NEIL3 & Forward & TGGAAGTGCAGCTCACCAAA \\
& Reverse & AGCACATCACCTAGCATCCG \\
$\beta$-actin & Forward & GTGCTATCCCTGTACGCCTC \\
& Reverse & AGGTAGTCAGTCAGGTCCCG \\
\hline
\end{tabular}

Cell invasion assay (chamber room method). Chamber rooms with $8-\mu \mathrm{m}$-pore size membranes (Corning Incorporated) were used to detect the invasion of liver cancer cells. Chamber rooms were pretreated with Matrigel (BD Biosciences) for $6 \mathrm{~h}$ at $37^{\circ} \mathrm{C}$ and $2 \times 10^{4}$ cells/well in DMEM with no serum were seeded into the upper chamber (the insert). In the bottom chamber (below the insert), DMEM with $10 \%$ FBS was added. After $24 \mathrm{~h}$ at $37^{\circ} \mathrm{C}$, cells on the top surface of the membrane of the insert were removed and cells in the bottom surface of the membrane of the insert were fixed in $4 \%$ paraformaldehyde at room temperature for $30 \mathrm{~min}$, washed and stained with $0.5 \%$ crystal violet at room temperature for $15 \mathrm{~min}$. Next, positively-stained cells were counted under a light inverted microscope ((NikonTS100; Nikon Corporation) at x100 magnification.

Distribution of the cell cycle (PI dying method). A total of $3 \times 10^{5}$ siNEIL3- or scramble-treated cells per well were seeded onto 6-well plates and cultured for $48 \mathrm{~h}$. Next, the cells were collected, washed and fixed in $75 \%$ cold alcohol for $24 \mathrm{~h}$ at $4^{\circ} \mathrm{C}$. Following centrifugation at $300 \mathrm{x}$ g for $5 \mathrm{~min}$ at room temperature and washing with cold PBS, $500 \mu \mathrm{l}$ staining buffer with $10 \mu \mathrm{l}$ PI and $10 \mu \mathrm{l}$ RNaseA solution (Shanghai Yeasen Biotechnology Co., Ltd.) was used to re-suspend the cells and cells were cultured at $37^{\circ} \mathrm{C}$ for $30 \mathrm{~min}$. Next, cells were analyzed using a flow cytometer (FACSCelesta; BD Biosciences). The edition number of software was BD FACSDiva Software v8.0.1.1.

Detection of cell apoptosis (Annexin V/FITC-PI dye). A total of $3 \times 10^{5}$ siNEIL3- or scramble-treated cells per well were seeded onto 6-well plates and cultured for $48 \mathrm{~h}$. Next, cells were collected, washed and stained using an Apoptosis Detection kit (Shanghai Yeasen Biotechnology Co., Ltd.), according to the manufacturer's protocols. In brief, staining buffer with $5 \mu 1$ FITC and $10 \mu 1$ PI solution was used to re-suspend cancer cells and samples were placed on ice for 15 min. Next, cell apoptosis was detected using a flow cytometer (FACSCelesta, BD Biosciences). The edition number of software was BD FACSDiva Software v8.0.1.1.

Statistical analysis. All data were statistically analyzed using SPSS 16.0 software (SPSS, Inc.).The unpaired Student's t-test method was used to evaluate the significance between two groups while the one-way analysis of variance method followed by Tukey's test was used to estimate the difference among multiple groups. All data are presented as the mean \pm standard deviation. ${ }^{*} \mathrm{P}<0.05$ was considered to indicate a statistically significant difference.
Table II. Clinical pathological analysis of NEIL3 expression with tumor grade.

\begin{tabular}{lc} 
Comparison & Statistical significance \\
\hline Normal $(\mathrm{n}=50)$ vs. grade $1(\mathrm{n}=54)$ & $7.57 \times 10^{-4}$ \\
Normal $(\mathrm{n}=50)$ vs. grade $2(\mathrm{n}=173)$ & $1.25 \times 10^{-12}$ \\
Normal $(\mathrm{n}=50)$ vs. grade $3(\mathrm{n}=118)$ & $2.67 \times 10^{-12}$ \\
Normal $(\mathrm{n}=50)$ vs. grade $4(\mathrm{n}=12)$ & $3.04 \times 10^{-3}$ \\
\hline
\end{tabular}

\section{Results}

NEIL3 is associated with short survival time in liver cancer. TCGA is a public database that consists of the clinical information of patients affected by cancer. Based on the TCGA data (URL:http://ualcan.path.uab.edu/cgi-bin/ualcan-res. $\mathrm{pl}$ ), the expression of the NEIL3 gene was analyzed in liver cancer tissues $(n=371)$ and normal control tissues $(n=50)$. As shown in Fig. 1A, the mean expression value of NEIL3 in liver cancer tissues was $\sim 28$ times as much as that in the normal control group. Clinicopathological analysis indicated that NEIL3 expression was correlated with tumor grade (Table II). However, no association was found with age, gender, race and weight. Next, by Kaplan-Meier method (32), patients with high NEIL3 expression $(\mathrm{n}=91)$ displayed poorer10-year survival probability than patients with low NEIL3 expression $(\mathrm{n}=274$; P $<0.001$; Fig. 1B). Therefore, NEIL3 was clinically associated with survival time and may serve important roles in liver cancer.

NEIL3 deficiency inhibits cell proliferation and growth in liver cancer. To investigate the role of NEIL3 in liver cancer, the expression of NEIL3 was detected in liver cancer cell lines. NEIL3 displayed much higher expression in HepG2 and Huh-7 cells than in the control group at both the mRNA and protein levels (Fig. 2A and B). Next, the mRNA expression of NEIL3 in HepG2 cells was decreased by RNAi technology. The knockdown efficiency was $\sim 80 \%$ in cells treated with siNEIL3-1 oligonucleotide (Fig. 2C and D). To detect the effect of NEIL3-knockdown on cell growth and proliferation, CCK-8 and plate-colony formation assays were performed. As shown in Fig. 3A, the cell proliferation rate decreased by $61.5 \%$ at $96 \mathrm{~h}$ after NEIL3 gene expression was decreased in HepG2 cells. The number of cell colonies decreased by $52.1 \%$ compared with the control (Fig. 3B and C). In Huh-7 cells, the cell proliferation rate decreased by $38 \%$ when the number of cell colonies decreased by $61.6 \%$ compared with the control (Fig. 4A-C). It is clear that NEIL3 contributes toward cell growth and proliferation in liver cancer.

NEIL3 deficiency suppresses cell migration and invasion in liver cancer. To detect the effects of NEIL3-knockdown on cell migration and invasion in liver cancer cells, wound-healing and Transwell assays were performed. As shown in Fig. $5 \mathrm{~A}$ and $\mathrm{B}$, the relative cell migration rate of HepG2 cells was decreased by $27 \%$ in NEIL3-knockdown cells, which suggested that siNEIL3 suppressed cell migration. The average cell number transferred through the chamber 

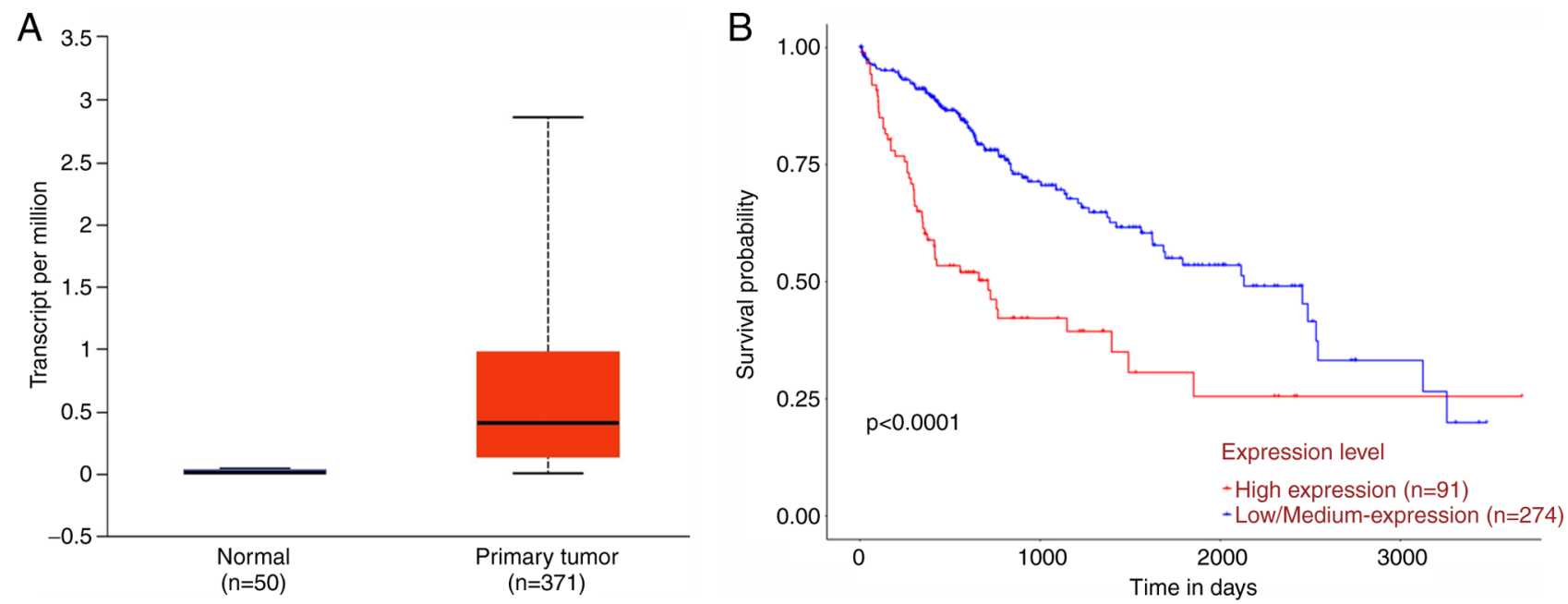

Figure 1. NEIL3 is overexpressed in liver cancer tissues and is associated with prognosis. (A) Based on TCGA data, NEIL3 was expressed significantly higher in tumor tissues compared with normal control tissues. (B) Survival curve of patients with liver cancer based on NEIL3 expression levels.

A

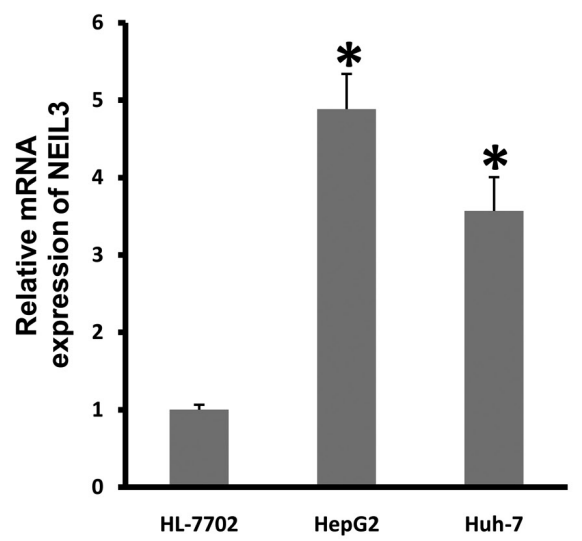

B

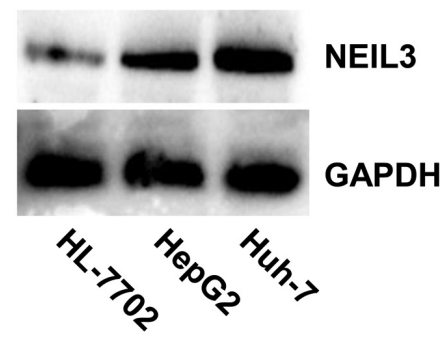

C

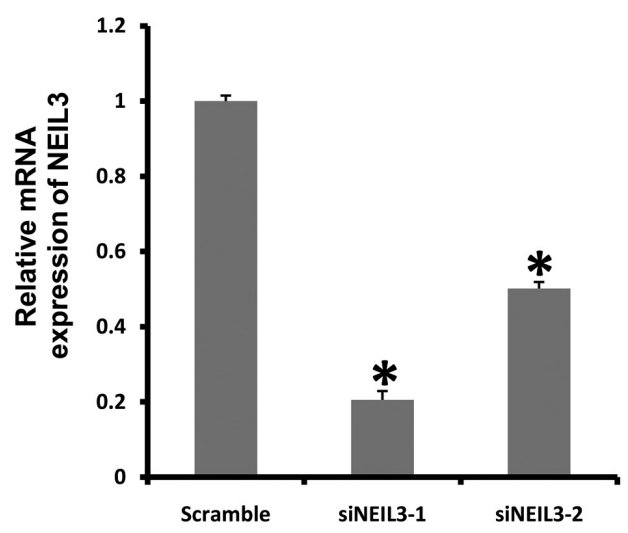

D

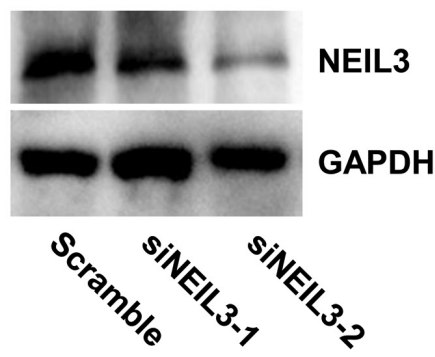

Figure 2.NEIL3 is knocked down in liver cancer cell lines. (A) The mRNA expression of NEIL3 in liver cancer cell lines by qPCR technology. (B) Protein level of NEIL3 in liver cancer cell lines by western blotting. (C) NEIL3 was knocked down at the mRNA level in HepG2 cells, as determined by qPCR technology. (D) NEIL3 was knocked down at the protein level in HepG2 cells, as determined by western blotting. ${ }^{*} \mathrm{P}<0.05$ indicates a statistically significant difference. qPCR, quantitative polymerase chain reaction; si, small interfering RNA.

membrane in siNEIL3-treated HepG2 cells was significantly lower than that in the scramble group (11 versus 52; $\mathrm{P}<0.05$; Fig. 5C and D). In Huh-7 cells, the relative cell migration rate in the NEIL3-knockdown group was $35.9 \%$ compared with the scramble group (Fig. 6A and B). The average number of Huh-7 cells transferred through the chamber membrane in the NEIL3-knockdown group was 53, compared with126 in the scramble control group (Fig. 6C and D). These data suggested that NEIL3 deficiency suppresses cell migration and invasion in liver cancer.

The cell cycle is affected by NEIL3 deficiency in liver cancer. The cell cycle is accelerated in cancer. FACS analysis with PI staining was performed to detect the effects of NEIL3 on cell cycle distribution in HepG2 and Huh-7 cells. The ratio of cells in the $\mathrm{G} 2$ phase was increased from 2.2 to $5.3 \%$ in the siNEIL3 


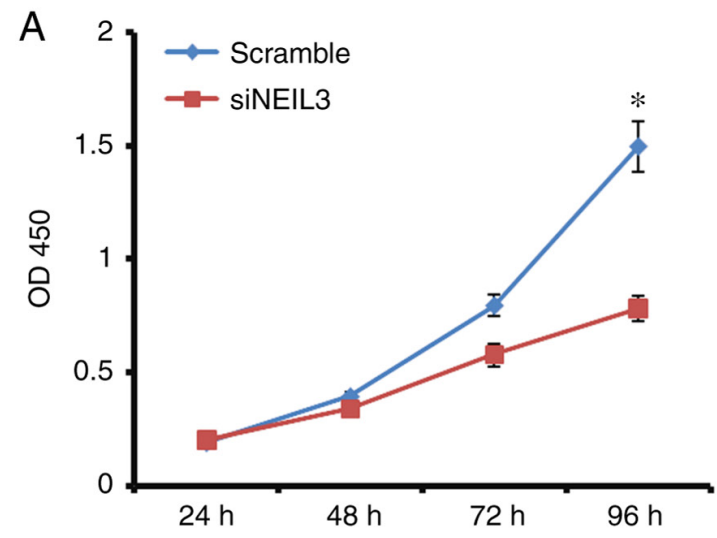

B
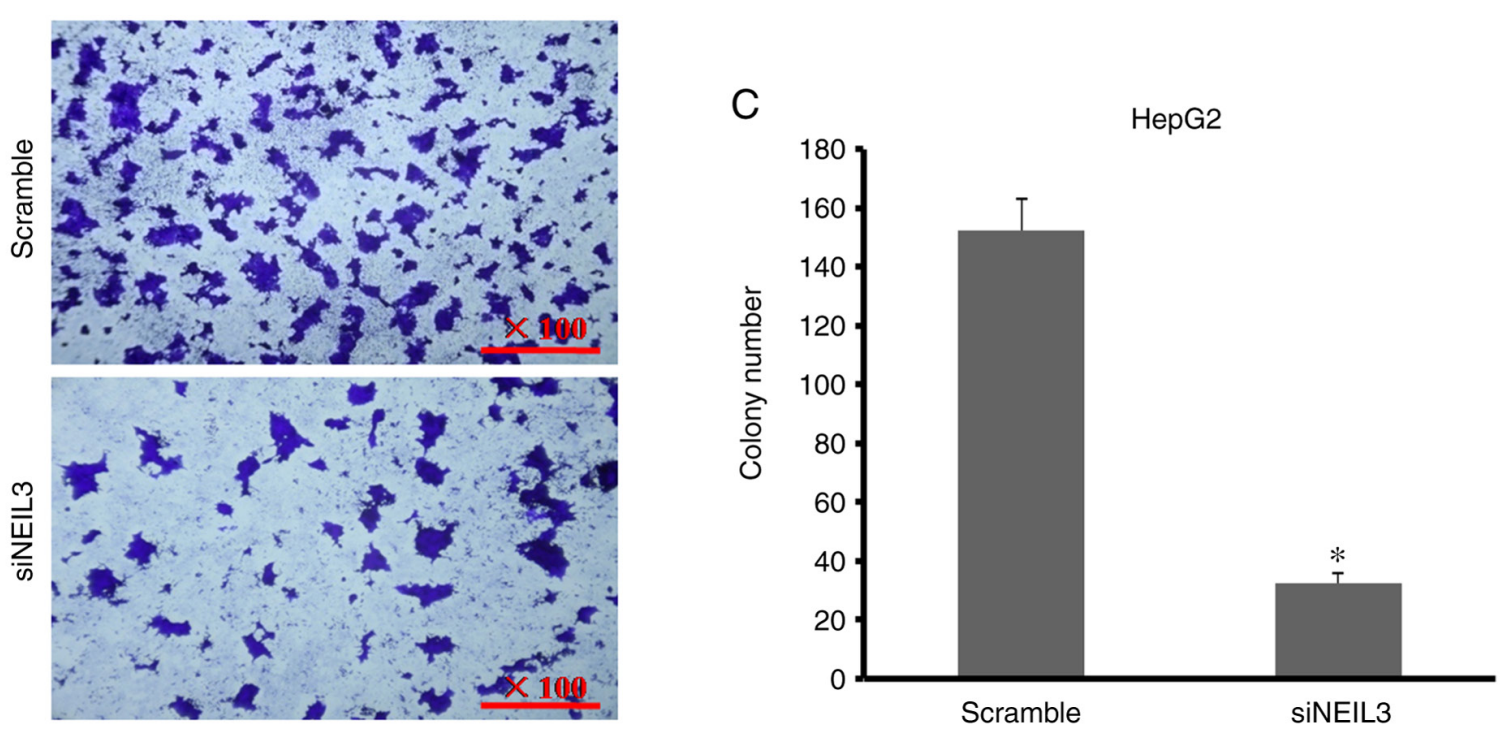

Figure 3. NEIL3 deficiency inhibits cell growth and proliferation in HepG2 cells. (A) Cell proliferation of HepG2 was inhibited following NEIL3 deficiency in a Cell Counting kit-8 assay. (B and C) Cell growth of HepG2 was inhibited following NEIL3 deficiency in a colony formation assay. ${ }^{\circ} \mathrm{P}<0.05$ indicates a statistically significant difference. OD, optical density; si, small interfering RNA.

group compared with the scramble control. The ratio of cells in the G1 and S phases was slightly decreased (Fig. 7A and B). In Huh-7 cells, cells in the G1 and S phases increased slightly, while cells in the G2 phase decreased slightly (Fig. 7C and D). However, none of the differences were significant $(\mathrm{P}>0.05)$, which suggests that the cell cycle is not the target of NEIL3 in liver cancer.

NEIL3 deficiency induces cell apoptosis in liver cancer. Suppression of programmed cell death (PCD) or cell apoptosis is adopted by nearly all tumors. By contrast, inducing cell apoptosis is the major mechanism of chemotherapy drugs in the clinic. Double-dye staining with Annexin V-FITC/PI solution was performed to detect the effect of siNEIL3 on cell apoptosis. In HepG2 cells, the mean apoptosis rate in the siNEIL3 group was 5.5\% following NEIL3-knockdown, but it was $0 \%$ in the scramble group (Fig. 8A and B). The difference between the two groups was significant $(\mathrm{P}<0.05)$. In Huh-7 cells, NEIL3-knockdown induced cell apoptosis ranging between 2.8 and $5.8 \%$ compared with the scramble control $(\mathrm{P}<0.05$; Fig. $8 \mathrm{C}$ and $\mathrm{D})$. Therefore, NEIL3 deficiency induces apoptosis in liver cancer.

NEIL3 regulates PI3K/Akt/mTOR signaling in liver cancer. NEIL3 was reported to regulate the expression of the
BRCA1/2oncogene in cancer (33). The present study reported that the phosphorylation level of PI3K (piPI3K) was decreased when NEIL3 was decreased in HepG2 cells (Fig. 9A and B). In addition, the phosphorylation levels of downstream molecules of the PI3K signaling pathway, including Akt (piAkt) and mTOR (pimTOR), were also decreased. By contrast, overexpression of NEIL3 enhanced the phosphorylation level of PI3K, Akt and mTOR in HepG2 cells. However, the background expression levels of PI3K, Akt and mTOR did not exhibit clear alterations regardless of NEIL3-knockdown in HepG2 cells (Fig. 9C). Therefore, NEIL3 at least partially regulates the activation of the PI3K/Akt/mTOR signaling pathway in liver cancer.

\section{Discussion}

Liver cancer is one of the ten most malignant cancer types in humans. Thousands of individuals die of liver cancer each year and the number of deaths keeps growing steadily (1). One major reason is the absence of knowledge of the cause and the molecular mechanism underlying liver cancer. In the clinic, liver cancer is heterogeneous, and patients display different responses to particular therapies or drugs (3). DNA sequencing technology suggests that genetic variation serves critical roles 


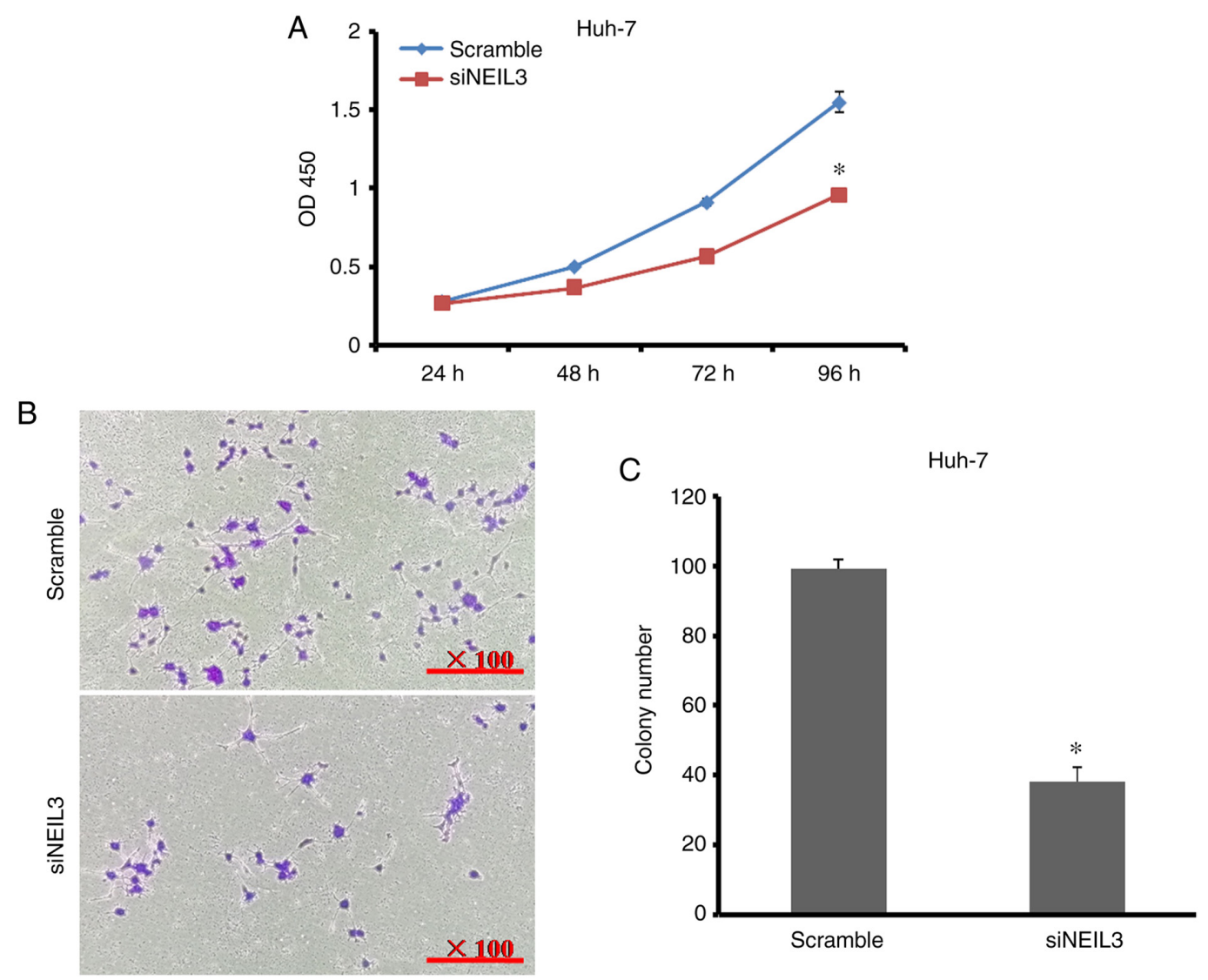

Figure 4. NEIL3 deficiency inhibits cell growth and proliferation in Huh-7 cells. (A) Cell proliferation of Huh-7 was inhibited following NEIL3 deficiency in a Cell Counting kit-8 assay. (B and C) Growth of Huh-7 cells was inhibited following NEIL3 deficiency in a colony formation assay. "P<0.05 indicates a statistically significant difference. OD, optical density; si, small interfering RNA.

A

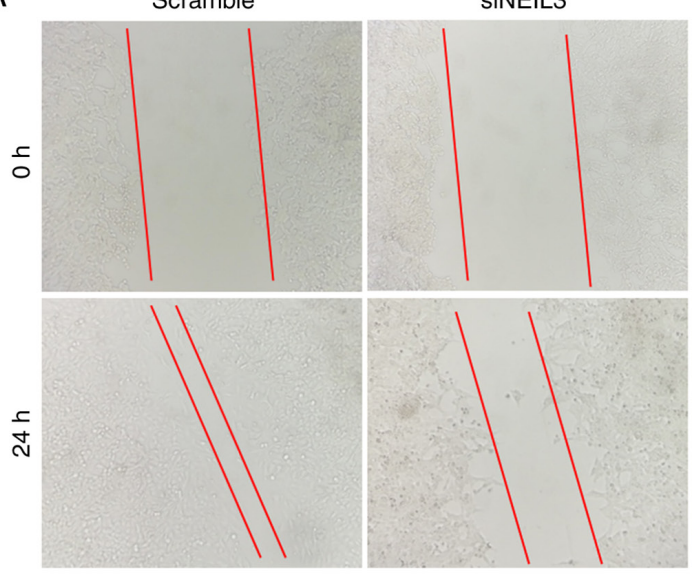

C

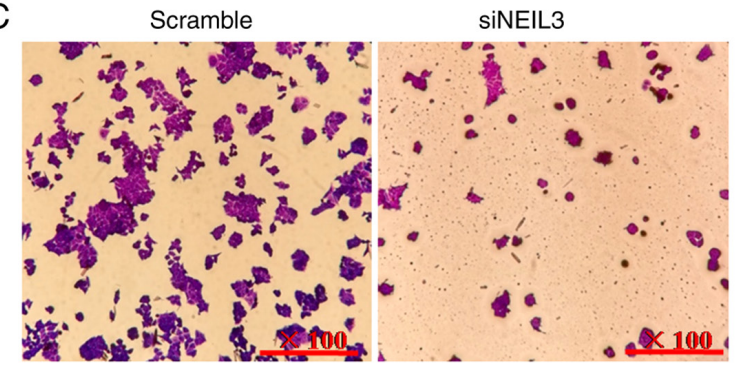

B

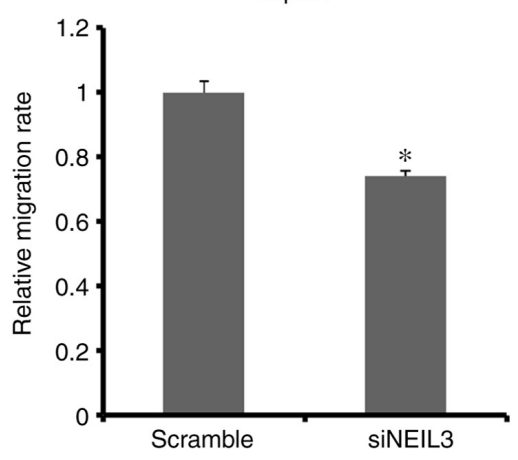

D

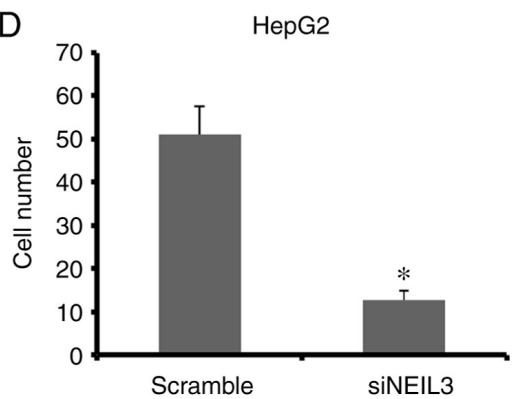

Figure 5. NEIL3 deficiency inhibits cell migration and invasion in HepG2 cells. (A and B) In a wound-healing assay, migration of HepG2 cells was inhibited in the NEIL3 deficiency group. (C and D) In a Transwell assay, invasion of HepG2 cells was inhibited in the NEIL3 deficiency group. ${ }^{*} \mathrm{P}<0.05$ indicates a statistically significant difference. si, small interfering RNA. 
A

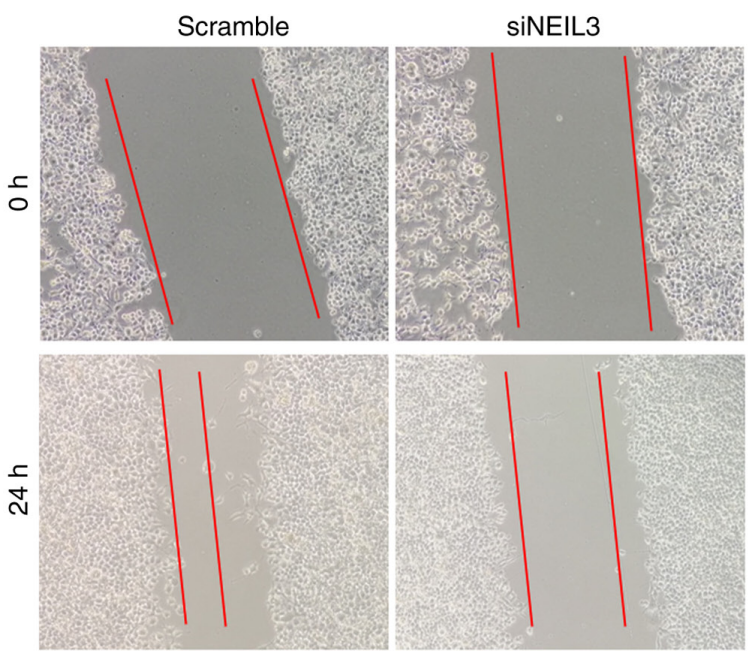

C

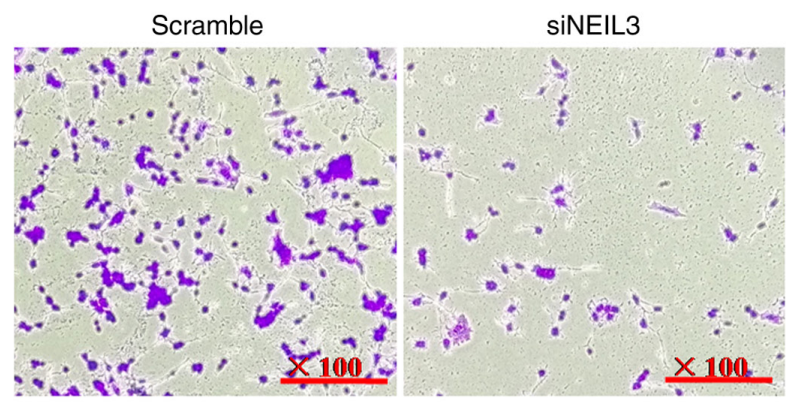

$\mathrm{B}$

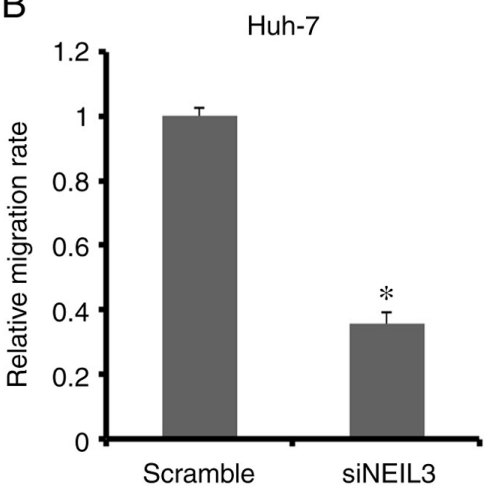

Huh-7

D

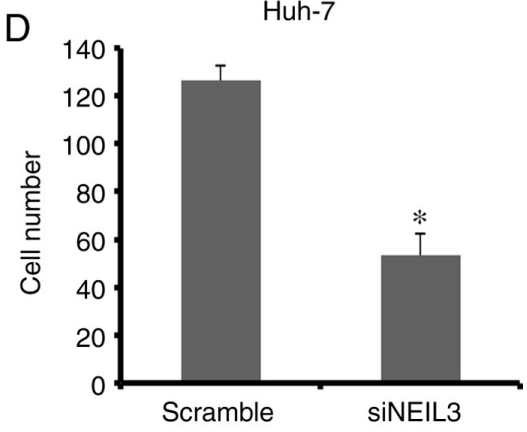

Figure 6. NEIL3 deficiency inhibits cell migration and invasion in Huh-7 cells. (A and B) In a wound-healing assay, migration of Huh-7 cells was inhibited in the NEIL3 deficiency group. (C and D) In a Transwell assay, invasion of Huh-7 cells was inhibited in the NEIL3 deficiency group. ${ }^{*} \mathrm{P}<0.05$ indicates a statistically significant difference. si, small interfering RNA.
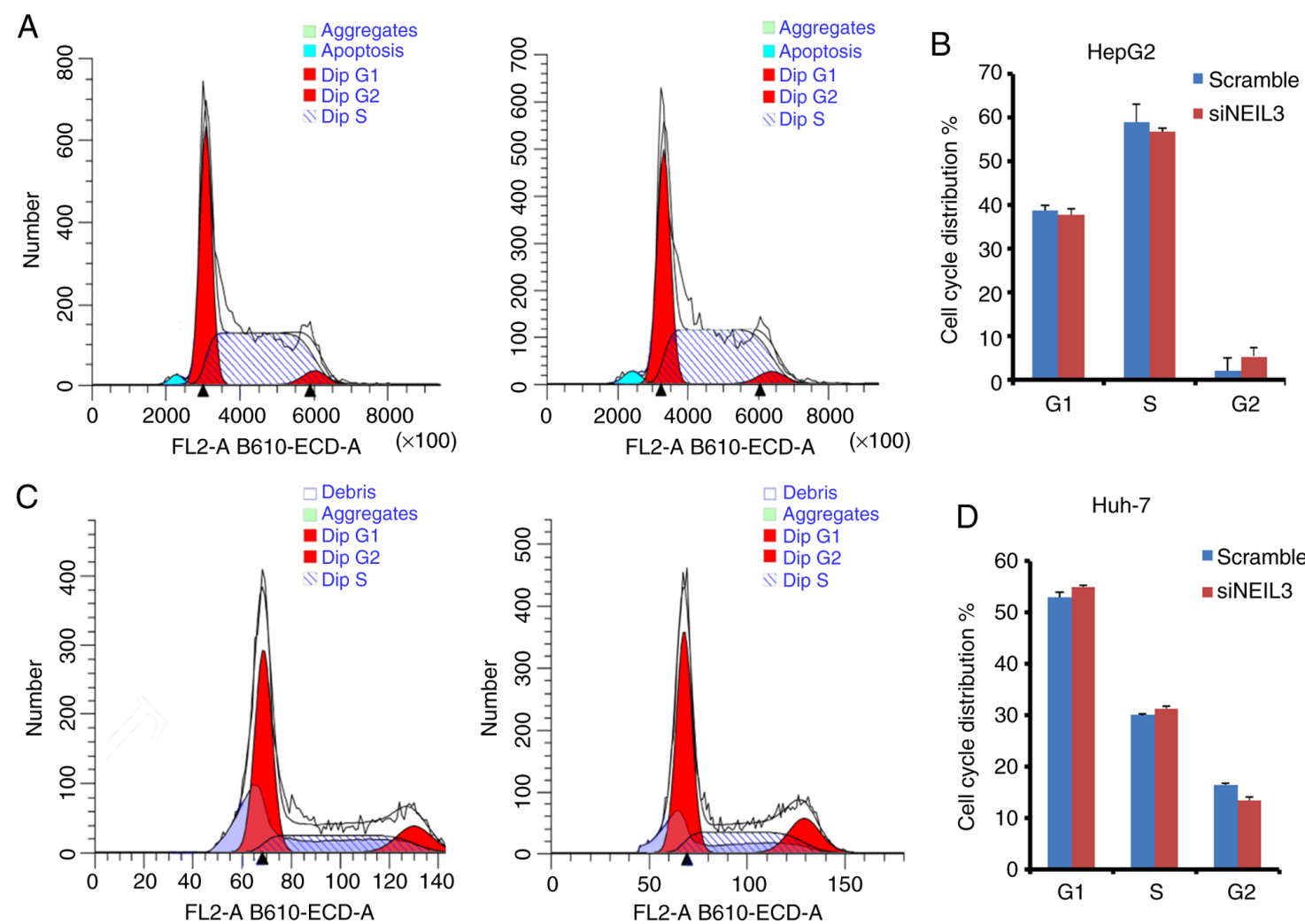

Figure 7. NEIL3 deficiency affects the cell cycle in liver cancer. (A) FACS analysis of the distribution of HepG2 cells in different phases in the context of NEIL3 deficiency. (B) Statistical analysis of data in A. (C) FACS analysis of the distribution of Huh-7 cells in different phases in the context of NEIL3 deficiency. (D) Statistical analysis of data in C. si, small interfering RNA. 

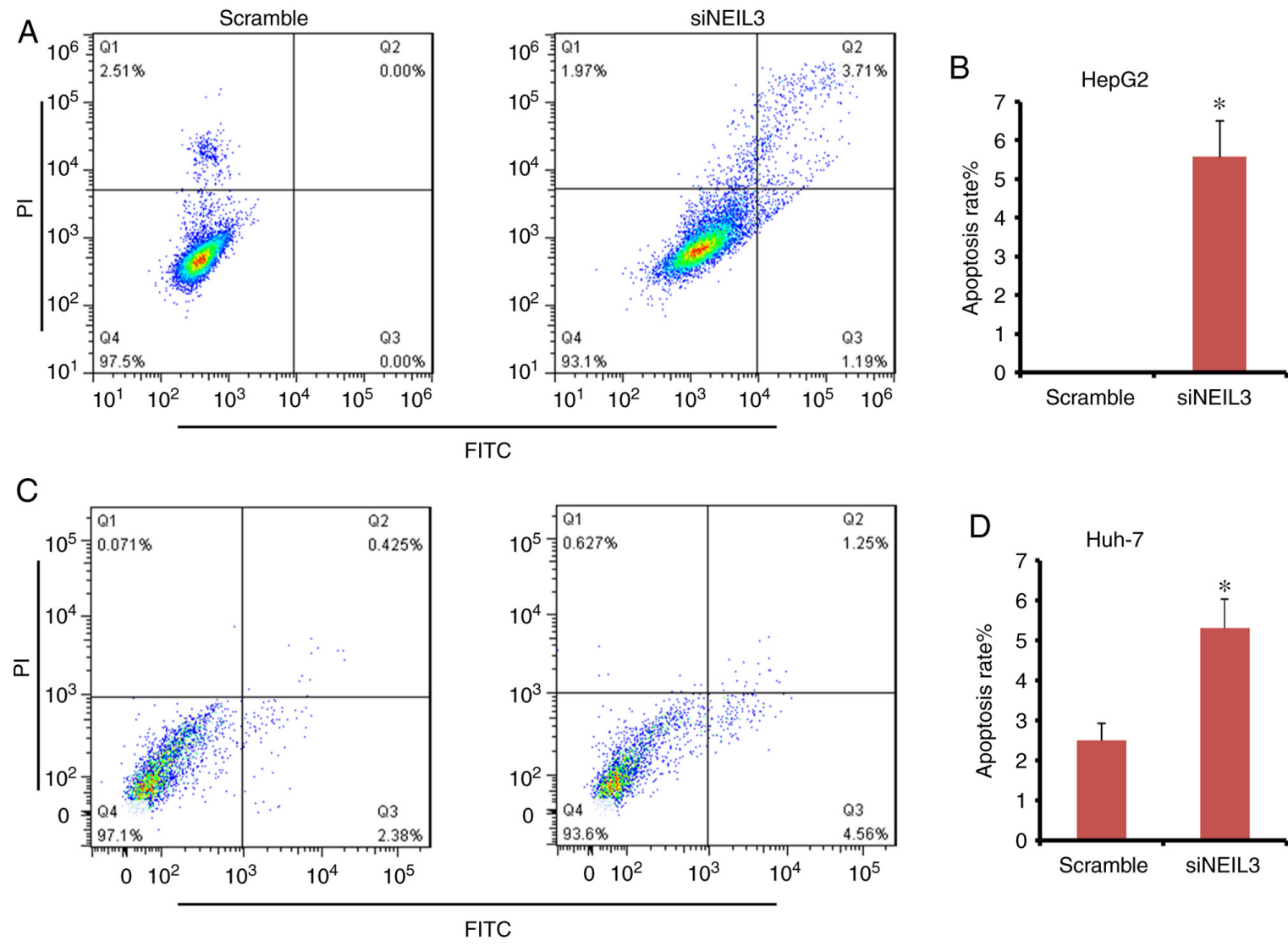

Figure 8. NEIL3 deficiency induces cell apoptosis in liver cancer. (A) FACS analysis of the ratio of HepG2 cells after NEIL3 expression was decreased. (B) Statistical analysis of data in A. (C) FACS analysis of the ratio of Huh-7 cells after NEIL3 expression was decreased. (D) Statistical analysis of data in C. ${ }^{*} \mathrm{P}<0.05$ indicates a statistically significant difference. si, small interfering RNA.

A

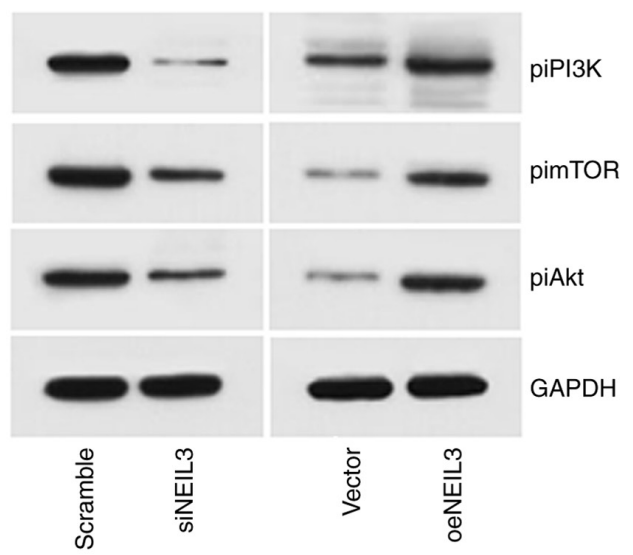

C

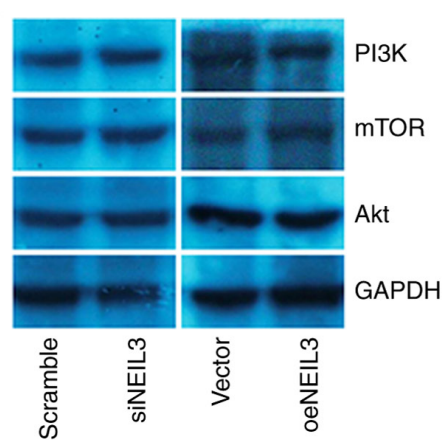

B
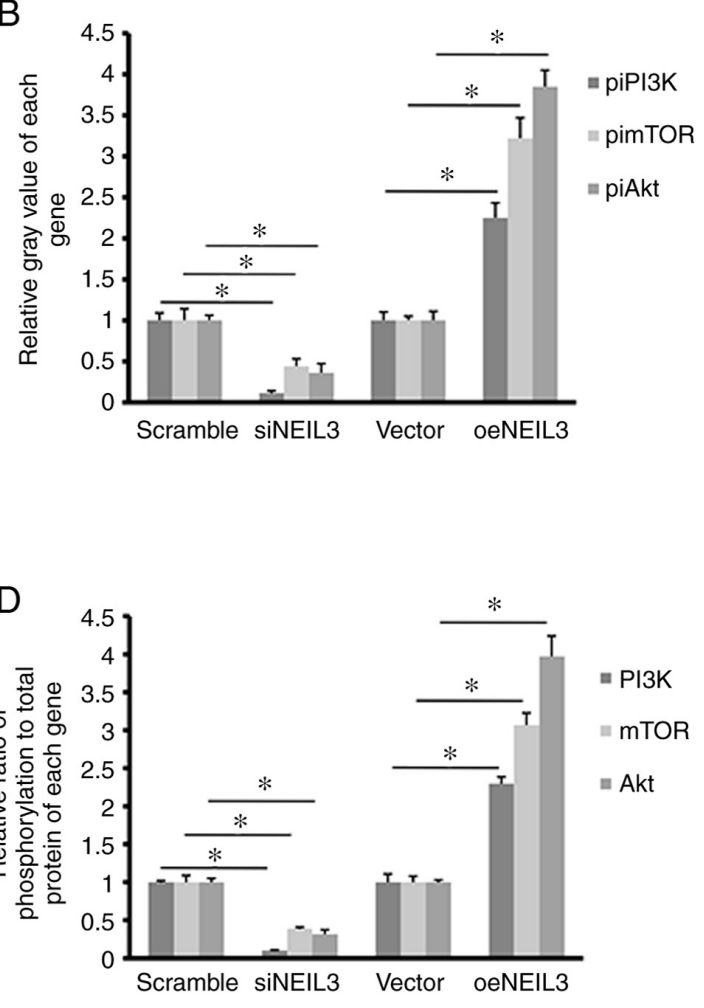

Figure 9. NEIL3 regulates the phosphorylation levels of PI3K/Akt/mTOR signaling molecules. (A) In western blotting, the phosphorylation level of Akt, PI3K and mTOR were regulated by NEIL3. (B) The gray value analysis of each band in A. (C) The background expression levels of Akt, PI3K and mTOR. (D) Relative ratio of phosphorylated protein to total protein of each gene. ${ }^{\mathrm{P}}<0.05$ indicates a statistically significant difference. si, small interfering RNA; oe, overexpression. 
in the formation and progression of liver cancer (34). Genetic variations, including gene mutations, alterations, truncations and fusions are major causes of heterogeneity in cancer and have been considered in precision medicine (35). For example, PD-L1 is a tumor antigen that is expressed on the surface of cancer cells (36). Opadivo, a specific antibody drug against PD-1, was developed and displayed great success in the clinic for the treatment of liver cancer (37). In fact, dozens of antibody drugs have been approved to treat cancer over recent years. However, genetic variations have prolonged the process to fight cancer.

TCGA is a public database containing a large quantity of information from patients with cancer and it has greatly advanced research in cancer (38). In the present study, by extracting the genetic data regarding liver cancer from TCGA, it was found that NEIL3 was overexpressed in patients with liver cancer and was associated with tumor stage. Furthermore, higher expression of NEIL3 predicted poorer survival, suggesting that NEIL3 may serve very important roles in liver cancer in the clinic. This was consistent with the role of NEIL3 in colorectal cancer and astrocytoma $(21,22)$. Indeed, a list of genes from TCGA database was proven to be involved in the formation and/or progression of liver cancer. An in vitro assay further supported the significance of NEIL3 in liver cancer. As described earlier, knockdown of NIEL3 inhibited cell growth, proliferation, migration, invasion and cell cycle transition, and induced cell apoptosis in HepG2 and Huh-7 cells. Cancer cells are characterized by potent abilities of proliferation and invasion, accelerated cell division and suppressed cell apoptosis (39). In physiological conditions, normal cells show contact-inhibitory activity and communicate with each other to survive in a limited environment (40). By contrast, cancer cells lost this limitation and evolve to have potent proliferation abilities, which results in unlimited tumor expansion (40). Accelerated cell life also contributes toward cell expansion. The entire life of a cell can be divided into G1, S, G2 and M phase. Additionally, cell cycle transition is controlled strictly by checkpoint mechanisms (41). However, cancer cells evolve a novel mechanism to circumvent the checkpoints and accelerate cell cycle transition, which promotes cell division (42). In the present study, NEIL3 affected cell cycle transition, but not significantly, suggesting that NEIL3mayregulate other behaviors in liver cancer. Apoptosis is an important mechanism for homeostasis but is suppressed in cancer $(43,44)$. NEIL3-knockdown in HepG2 and Huh-7 cells induced apoptosis, which suggested that NEIL3 was a negative factor for apoptosis in liver cancer. This finding further suggests that NEIL3 promotes progression in liver cancer. NEIL3 was also reported to promote progression in breast, ovarian and prostate cancer (23-25). As a result, it is hypothesized that NEIL3 contributes toward the development and/or progression of liver cancer.

The PI3K/Akt and mTOR signaling pathways serve important roles in normal development and regulate a variety of physiological processes, including cell proliferation, migration, survival and differentiation (45). The PI3K family consists of heterodimeric lipid kinases and maybe classified into three classes based on substrate specificity and sequence homology (46). Of them, class 1 PI3Ks are responsible for catalyzing PIP2 into the secondary messenger, PIP3, which recruits Akt to the inner membrane and activates Akt by phosphorylating its serine/threonine kinase sites (46). Tuberous sclerosis complex (TSC) negatively regulates the activation of mTORC1 signaling, while activated Akt inhibits TSC complex activity and initiates the mTOR signaling pathway followed by phosphorylated activation of eIF4E and 4EBP1 $(47,48)$. Next, cell growth and proliferation are switched on. However, abnormal activation of PI3K/Akt/mTOR signaling often leads to severe diseases, including cancer. PI3K/Akt/mTOR signaling shows markedly increased activity in cancer. For example, Chen and Costa (49) reported that activation of $\mathrm{PI} 3 \mathrm{~K} / \mathrm{Akt} / \mathrm{mTOR}$ signaling caused a number of cancer types. In glioblastoma, inhibitors targeting PI3K/Akt/mTOR signaling provided a promising way to fight the disease (50). In liver cancer, the PI3K/Akt/mTOR signaling pathway was a promising target for screening effective drugs (51). The present study proved, by western blotting, that NEIL3 mayregulate the phosphorylation levels of PI3K, Akt and mTOR, which suggests that NEIL3 regulates the PI3K/Akt/mTOR signaling pathway in liver cancer. However, further investigation is necessary to confirm this. Furthermore, the clinical significance of NEIL3 in liver cancer needs to be evaluated in a large cohort of patients.

In summary, NEIL3 contributes toward cell growth, proliferation, migration and invasion, but inhibits apoptosis in liver cancer. NEIL3 regulates $\mathrm{PI} 3 \mathrm{~K} / \mathrm{Akt} / \mathrm{mTOR}$ signaling in liver cancer and is associated with prognosis in patients with liver cancer.

\section{Acknowledgements}

Not applicable.

\section{Funding}

No funding was received.

\section{Availability of data and materials}

The datasets used and/or analyzed during the current study are available from the corresponding author on reasonable request.

\section{Authors' contributions}

WW performed the main experiments and wrote the manuscript. QY analyzed and interpreted the data from TCGA database and in the experiment. SG performed part of the experiment and analyzed the experiment data. JW designed the whole study and reviewed the manuscript. All authors read and approved the final manuscript.

\section{Ethics approval and consent to participate}

Not applicable.

\section{Patient consent for publication}

Not applicable.

\section{Competing interests}

The authors declare that they have no competing interests. 


\section{References}

1. Siegel RL, Miller KD and Jemal A: Cancer Statistics, 2019. CA Cancer J Clin 69: 7-34, 2019.

2. Liu CY, Chen KF and Chen PJ: Treatment of liver cancer. Cold Spring Harb Perspect Med 5: a021535, 2015.

3. Li L and Wang H: Heterogeneity of liver cancer and personalized therapy. Cancer Lett 379: 191-197, 2016.

4. Marin JJG, Cives-Losada C, Asensio M, Lozano E, Briz O and Macias RIR: Mechanisms of anticancer drug resistance in hepatoblastoma. Cancer (Basel) 11: 407, 2019.

5. Keating GM: Sorafenib: A review in hepatocellular carcinoma Target Oncol 12: 243-253, 2017.

6. Zhu YJ, Zheng B, Wang HY and Chen L: New knowledge of the mechanisms of sorafenib resistance in liver cancer. Acta Pharmacol Sin 38: 614-622, 2017.

7. Liu MM, Bandaru V, Bond JP, Jaruga P, Zhao X, Christov PP, Burrows CJ, Rizzo CJ, Dizdaroglu M and Wallace SS: The mouse ortholog of NEIL3 is a functional DNA glycosylase in vitro and in vivo. Proc Natl Acad Sci USA 107: 4925-4930, 2010.

8. Bandaru V, Sunkara S, Wallace SS and Bond JP: A novel human DNA glycosylase that removes oxidative DNA damage and is homologous to Escherichia coli endonuclease VIII. DNA Repair (Amst) 1: 517-529, 2002.

9. Liu M, Doublie S and Wallace SS: Neil3, the final frontier for the DNA glycosylases that recognize oxidative damage. Mutat Res 743-744: 4-11, 2013.

10. Jalland CM, Scheffler K, Benestad SL, Moldal T, Ersdal C, Gunnes G, Suganthan R, Bjørås M and Tranulis MA: Neil3 induced neurogenesis protects against prion disease during the clinical phase. Sci Rep 6: 37844, 2016.

11. Sejersted Y, Hildrestrand GA, Kunke D, Rolseth V, Krokeide SZ, Neurauter CG, Suganthan R, Atneosen-Åsegg M, Fleming AM, Saugstad OD, et al: Endonuclease VIII-like 3 (Neil3) DNA glycosylase promotes neurogenesis induced by hypoxia-ischemia. Proc Natl Acad Sci USA 108: 18802-18807, 2011.

12. Regnell CE, Hildrestrand GA, Sejersted Y,Medin T, Moldestad O, Rolseth V, Krokeide SZ, Suganthan R, Luna L, Bjørås M and Bergersen LH: Hippocampal adult neurogenesis is maintained by Neil3-dependent repair of oxidative DNA lesions in neura progenitor cells. Cell Rep 2: 503-510, 2012.

13. Skarpengland T, Holm S, Scheffler K, Gregersen I, Dahl TB Suganthan R, Segers FM, Østlie I, Otten JJ, Luna L, et al: Neil3-dependent base excision repair regulates lipid metabolism and prevents atherosclerosis in Apoe-deficient mice. Sci Rep 6 : 28337, 2016

14. Yang LX, Zhang X and Zhao G: Ginsenoside Rd attenuates DNA damage by increasing expression of DNA glycosylase endonuclease VIII-like proteins after focal cerebral ischemia. Chin Med J (Engl) 129: 1955-1962, 2016.

15. Massaad MJ, Zhou J, Tsuchimoto D, Chou J, Jabara H, Janssen E, Glauzy S, Olson BG, Morbach H, Ohsumi TK, et al: Deficiency of base excision repair enzyme NEIL3 drives increased predisposition to autoimmunity. J Clin Invest 126: 4219-4236, 2016.

16. Smatlikova P, Askeland G, Vaskovicova M, Klima J, Motlik J, Eide L and Ellederová Z: Age-related oxidative changes in primary porcine fibroblasts expressing mutated huntingtin. Neurodegener Dis 19: 22-34, 2019.

17. Zhou H, Xu M, Huang Q, Gates AT, Zhang XD, Castle JC, Stec E, Ferrer M, Strulovici B, Hazuda DJ and Espeseth AS Genome-scale RNAi screen for host factors required for HIV replication. Cell Host Microbe 4: 495-504, 2008.

18. Shinmura K, Kato H, Kawanishi Y, Igarashi H, Goto M, Tao H, Inoue $\mathrm{Y}$, Nakamura S, Misawa $\mathrm{K}$, Mineta $\mathrm{H}$ and Sugimura $\mathrm{H}$ : Abnormal expressions of DNA glycosylase genes NEIL1, NEIL2, and NEIL3 are associated with somatic mutation loads in human cancer. Oxid Med Cell Longev 2016: 1546392, 2016.

19. Kim YS, Kim Y, Choi JW, Oh HE and Lee JH: Genetic variants and risk of prostate cancer using pathway analysis of a genome-wide association study. Neoplasma 63: 629-634, 2016.

20. Klattenhoff AW, Thakur M, Chu CS, Ray D, Habib SL and Kidane D: Loss of NEIL3 DNA glycosylase markedly increases replication associated double strand breaks and enhances sensitivity to ATR inhibitor in glioblastoma cells. Oncotarget 8 : 112942-112958, 2017.

21. Jiraskova K, Hughes DJ, Brezina S, Gumpenberger $T$, Veskrnova V, Buchler T, Schneiderova M, Levy M, Liska V, Vodenkova S, et al: Functional polymorphisms in DNA repair genes are associated with sporadic colorectal cancer susceptibility and clinical outcome. Int J Mol Sci 20: 97, 2018.
22. de Sousa JF, Torrieri R, Searfim RB, Di Cristofaro LF, Escanfella DF, Ribeiro R, Zanette DL, Paçó-Larson ML, da Silva WA Jr, Tirapelli DP, et al: Expression signatures of DNA repair genes correlate with survival prognosis of astrocytoma patients. Tumour Biol 39: 1010428317694552, 2017.

23. Matta J, Morales L, Dutil J, Bayona M, Alvarez C and Suarez E: Differential expression of DNA repair genes in Hispanic women with breast cancer. Mol Cancer Biol 1: 54, 2013.

24. Nwani NG, Condello S, Wang Y, Swetzig WM, Barber E, Hurley T and Matei D: A novel ALDH1A1 inhibitor targets cells with stem cell characteristics in ovarian cancer. Cancers (Basel) 11: 502, 2019.

25. Barry KH, Koutros S, Berndt SI, Andreotti G, Hoppin JA, Sandler DP, Burdette LA, Yeager M, Freeman LE, Lubin JH, et al: Genetic variation in base excision repair pathway genes, pesticide exposure, and prostate cancer risk. Environ Health Perspect 119: 1726-1732, 2011

26. Zhou J, Chan J, Lambele M, Yusufzai T, Stumpff J, Opresko PL, Thali $\mathrm{M}$ and Wallace SS: NEIL3 repairs telomere damage during $\mathrm{S}$ phase to secure chromosome segregation at mitosis. Cell Rep 20: 2044-2056, 2017.

27. McNally EJ, Luncsford PJ and Armanios M: Long telomeres and cancer risk: The price of cellular immortality. J Clin Invest 130: 3474-3481, 2019

28. Chen X, Tang WJ, Shi JB, Liu MM and Liu XH: Therapeutic strategies for targeting telomerase in cancer. Med Res Rev 40: 532-585, 2020.

29. Zhang H, Ma H, Wang Q, Chen M, Weng D, Wang H, Zhou J, Li Y, Sun J, Chen Y, et al: Analysis of loss of heterozygosity on chromosome $4 \mathrm{q}$ in hepatocellular carcinoma using high-throughput SNP array. Oncol Rep 23: 445-455, 2010.

30. Masters JR, Thomson JA, Daly-Burns B, Reid YA, Dirks WG, Packer P, Toji LH, Ohno T, Tanabe H, Arlett CF, et al: Short tanderm repeat profiling provides an international reference standard for human cell lines. Proc Natl Acad Sci USA 98 8012-8017, 2001

31. Livak KJ and Schmittgen TD: Analysis of relative gene expression data using real-time quantitative PCR and the 2(-Delta Delta C(T)) method. Methods 25: 402-408, 2001

32. Bland JM and Altman DG: Survival probabilities (the Kaplan-Meier method). BMJ 317: 1572, 1998.

33. Tran OT, Tadesse S, Chu C and Kidane D: Overexpression of NEIL3 associated with altered genome and poor survival in selected types of human cancer. Tumour Biol 42: $1010428320918404,2020$.

34. Fujimoto A, Furuta M, Totoki Y, Tsunoda T, Kato M, Shiraishi Y, Tanaka H, Taniguchi H, Kawakami Y, Ueno M, et al: Whole-genome mutational landscape and characterization of noncoding and structural mutations in liver cancer. Nat Genet 48 : 500-509, 2016

35. Ma G, Yang X, Liang Y, Wang L, Li D, Chen Y, Liang Z, Wang Y and Niu H: Precision medicine and bladder cancer heterogeneity. Bull Cancer 105: 925-931, 2018.

36. Constantinidou A, Alifieris C and Trafalis DT: Targeting programmed cell death-1 (PD-1) and ligand (PD-L1): A new era in cancer active immunotherapy. Pharmacol Ther 194: 84-106, 2019.

37. Tella SH, Mahipal A, Kommalapati A and Jin Z: Evaluating the safety and efficacy of Nivolumab in patients with advanced hepatocellular carcinoma: Evidence to date. Onco Targets Ther 12: 10335-10342, 2019.

38. Blum A, Wang P and Zenklusen JC: SnapShot: TCGA-analyzed tumors. Cell 173: 530, 2018

39. Fouad YA and Aanei C: Revisiting the hallmarks of cancer. Am J Cancer Res 7: 1016-1036, 2017

40. Ribatti D: A revisited concept: Contact inhibition of growth. From cell biology to malignancy. Exp Cell Res 359: 17-19, 2017.

41. Barnum KJ and Q'Connell MJ: Cell cycle regulation by checkpoints. Methods Mol Biol 1170: 29-40, 2014.

42. Wenzel ES and Singh ATK: Cell-cycle checkpoints and aneuploidy on the path to cancer. In Vivo 32: 1-5, 2018.

43. Tuzlak S, Kaufmann T and Villunger A: Interrogating the relevance of mitochondrial apoptosis for vertebrate development and postnatal tissue homeostasis. Genes Dev 30: 2133-2151, 2016.

44. Goldar S, Khaniani MS, Derakhshan SM and Baradaran B: Molecular mechanisms of apoptosis and roles in cancer development and treatment. Asian Pac J Cancer Prev 16: 2129-2144, 2015.

45. Engelman JA, Luo J and Cantley LC: The evolution of phosphatidylinositol 3-kinases as regulators of growth and metabolism. Nat Rev Genet 7: 606-619, 2006. 
46. Czech MP: PIP2 and PIP3: Complex roles at the cell surface. Cell 100: 603-606, 2000.

47. Dowling RJ, Topisirovic I, Fonseca DB and Sonenberg N: Dissecting the role of mTOR: Lessons from mTOR inhibitors. Biochim Biophys Acta 1804: 433-439, 2010.

48. Hay N and Sonenberg N: Upstream and downstream of mTOR. Genes Dev 18: 1926-1945, 2014.

49. Chen QY and Costa M: PI3K/Akt/mTOR signaling pathway and the biphasic effect of arsenic in carcinogenesis. Mol Pharmacol 94: 784-792, 2018.

50. Li X, Wu C, Chen N, Gu H, Yen A, Cao L, Wang E and Wang L: $\mathrm{PI} 3 \mathrm{~K} / \mathrm{Akt} / \mathrm{mTOR}$ signaling pathway and targeted therapy for glioblastoma. Oncotarget 7: 33440-33450, 2016.
51. Kattan SW, Nafie MS, Elmgeed GA, Alelwani W, Badar M and Tantawy MA: Molecular docking, anti-proliferative activity and induction of apoptosis in human liver cancer cells treated with androstane derivatives: Implication of PI3K/AKT/mTOR pathway. J Steroid Biochem Mol Biol 198: 105604, 2020.

(i) () $९$ This work is licensed under a Creative Commons Attribution-NonCommercial-NoDerivatives 4.0 International (CC BY-NC-ND 4.0) License. 